\title{
ORIENTATION ANGLE ESTIMATION OVER FORESTED TERRAIN USING P-BAND POLSAR DATA
}

\author{
Stefan Sauer, Thomas Jagdhuber, Florian Kugler, Seung-Kuk Lee, Kostas Papathanassiou
}

German Aerospace Center (DLR), Microwaves and Radar Institute (HR), Germany, stefan.sauer@dlr.de

\begin{abstract}
One important secondary objective of the proposed EarthExplorer Candidate Mission BIOMASS is the retrieval of a digital terrain model (DTM) using satellite-borne P-band SAR data. The interferometric phase acquired in repeat-pass mode may be affected by ionospheric effects leading to corrupted phase estimates and, consequently, to errors in the derived DTM. In contrast, line-of-sight orientation angles induced by azimuth slopes can be estimated using single-pass POLSAR measurements. The orientation angle estimates allow a necessary pre-processing of POLSAR observations and the retrieval of topographic information such as a DTM. In this study, the performance of the circular polarization method for orientation angle estimation is examined over forested areas. To this end, the orientation angles computed from P-band POLSAR data are compared with the results obtained from LIDAR DTMs. In particular, the estimation performance is investigated with respect to the impact of topography and vegetation. POLSAR data at P-band are used that have been acquired by the E-SAR system of DLR over three forested test sites: two boreal forests in Sweden (one over flat terrain located at Remningstorp, the other over terrain with topographic variations at Krycklan) and the tropical forest Mawas in Indonesia over flat terrain.
\end{abstract}

Index Terms - POLSAR orientation angle estimation, forested terrain, vegetation bias.

\section{INTRODUCTION}

The main objective of the proposed Earth-Explorer Candidate BIOMASS Mission [1,2] is the improved mapping of global biomass. One of its secondary objectives is the estimation of a digital terrain model (DTM) employing P-band SAR data. As the BIOMASS mission is designed in a repeatpass interferometric mode, the interferometric phase may be influenced by the ionosphere [3] resulting in perturbed phase estimates and, consequently, in errors in the derived DTM. Alternatively, topographic information such as a DTM can be derived by computing the line-of-sight orientation angles from single-pass POLSAR data.

The estimation of line-of-sight orientation angles $[4,5]$ induced by azimuth slopes using polarimetric SAR (POLSAR) data has various application areas: First, after estimating the orientation angle, the POLSAR data can be compensated for this effect, which is a necessary pre-processing step to improve the quantitative retrieval of bio- and geophysical parameters [4]. The derived orientation angle can be used to retrieve the topography of the imaged terrain $[6,7]$ and generate a digital elevation model (DEM). The orientation angle can be also estimated in urban areas [8] where polarization orientation shifts are induced by tilted roofs and vertical building walls that are not aligned along the sensor trajectory.

In this paper, the performance of the circular polarization method for orientation angle estimation is investigated over forested terrain. For this purpose, the results obtained from Pband POLSAR data are compared with the orientation angles derived from LIDAR DTMs. In particular, the performance is examined with respect to topographic effects and vegetation bias. In this study, POLSAR data at P-band are employed that have been acquired by the E-SAR system of DLR over two boreal forests in Sweden (one over flat terrain, the other over terrain with topographic variations) and tropical forest in Indonesia over flat terrain.

\section{ORIENTATION ANGLE ESTIMATION}

The orientation angle generated by azimuth slopes can be written as [4]

$$
\tan \theta=\frac{\tan \omega}{-\tan \gamma \cos \phi+\sin \phi}
$$

where $\theta$ represents the orientation angle shift, $\omega$ the azimuth slope angle, $\gamma$ the slope in the ground range direction, and $\phi$ the radar look angle.

Relation (1) can be used to estimate the orientation angle from a DTM generated by LIDAR or InSAR data by computing the slope angles in azimuth and range directions.

To estimate the orientation angle from POLSAR data by the circular polarization method, the right-right and left-left circular polarizations are given by [4]

$$
\begin{aligned}
S_{R R} & =\left(S_{H H}-S_{V V}+i 2 S_{H V}\right) / 2 \\
S_{L L} & =\left(S_{V V}-S_{H H}+i 2 S_{H V}\right) / 2 .
\end{aligned}
$$

The orientation angle is derived as

$$
\theta=\left[\operatorname{Arg}\left(\left\langle S_{R R} S_{L L}^{*}\right\rangle\right)+\pi\right] / 4
$$


where Arg is the arctangent. To take into account negative orientation angles, $\theta$ has to be modified using the following rule [4]

$$
\text { For } \theta>\pi / 4 \text { replace } \theta \text { by }(\theta-\pi / 2) \text {. }
$$

The arctangent of the correlation between right-right and leftleft circular polarizations can be expressed as [4]

$$
\begin{aligned}
& \operatorname{Arg}\left(\left\langle S_{R R} S_{L L}^{*}\right\rangle\right)= \\
& \tan ^{-1}\left(\frac{-4 \operatorname{Re}\left(\left\langle\left(S_{H H}-S_{V V}\right) S_{H V}^{*}\right\rangle\right)}{-\left\langle\left|S_{H H}-S_{V V}\right|^{2}\right\rangle+4\left\langle\left|S_{H V}\right|^{2}\right\rangle}\right)
\end{aligned}
$$

where $\operatorname{Re}(x)$ represents the real part of $x$. The unambigiously measurable range of the orientation angle is limited between $-45^{0}$ and $45^{0}[4]$.

\section{RESULTS AND PERFORMANCE ANALYSIS}

\subsection{Boreal Forest Over Flat Terrain}

The first test site includes a hemiboreal forest over almost flat terrain and is located in Remningstorp, Southern Sweden. Figure 1 shows the Pauli color-coded image of the scene including mainly forested areas, but also some agricultural fields and lakes.

Using a boxcar filter of the size $20 \times 20$, the orientation angle has been estimated by the circular polarization method. As anticipated, the orientation angle estimate (see Figure 2 on the left hand side) is centered at around 0 deg, i.e., giving good estimation results, but is affected by noise.

To examine in detail the influence of vegetation and topography on the POLSAR orientation angle estimate, the parameter $\alpha_{\hat{\theta}_{P O L}}$ is defined as

$$
\alpha_{\hat{\theta}_{P O L}}=\left|\left\langle\exp \left(i 4 \hat{\theta}_{P O L}\right)\right\rangle\right| .
$$

It is a measure for the variations of the POLSAR-derived orientation angle where a low value near 0 corresponds to large variations and a high value near 1 to negligible variations.

It can be observed in Figure 2 on the right hand side that the noisy phase estimates are related to areas of large variations of the POLSAR-derived orientation angle. As depicted in the histogram of the variation parameter $\alpha_{\hat{\theta}_{P O L}}$ in Figure 3 on the left hand side, most of the samples possess negligible variations of the POLSAR-derived orientation angle with values greater than 0.95 .

The RMSE over the whole image is at 7.2 deg with a bias of $-0.4 \mathrm{deg}$. The dependence of the RMSE on the POLSARderived orientation angle variation has been investigated by taking into account only the samples above a given threshold thrsh, i.e., with

$$
\alpha_{\hat{\theta}_{P O L}} \geq \text { thrsh. }
$$

Increasing the threshold from 0 to $\frac{149}{151}$ (Figure 3 on the right hand side), the RMSE decreases to approximately 3 deg indicating that the orientation angle estimate improves if it does not vary significantly.

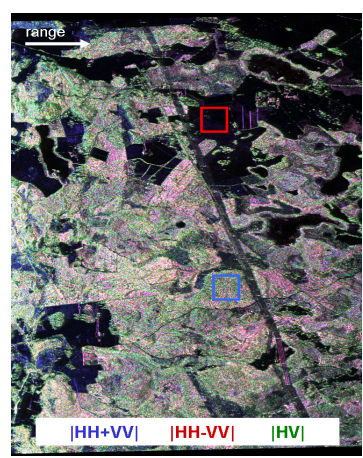

Fig. 1. Pauli color-coded image of the test site of Remningstorp.

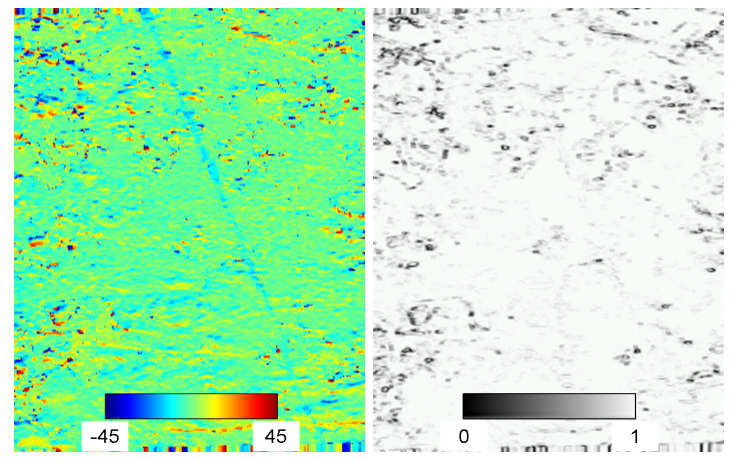

Fig. 2. Left: POLSAR-derived orientation angle over the test site of Remningstorp. Right: Variations of the POLSARderived orientation angle $\alpha_{\hat{\theta}_{P O L}}$.

To investigate the influence of the vegetation on the orientation angle estimation performance, a homogeneous area consisting of bare soil has been selected as shown in Figure 1 by the red square. For this cut-out, the RMSE reduces to around $3.7 \mathrm{deg}$ with a bias of $-0.5 \mathrm{deg}$. Comparable accuracy can be achieved over forested areas with negligible orientation angle variations (e.g., blue square in Figure 1) with RMSE of approximately 4 deg and slightly higher bias of around $-1.5 \mathrm{deg}$.
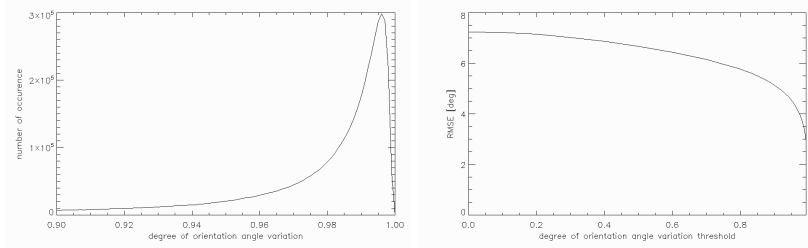

Fig. 3. Left: Histogram of variations of the POLSAR-derived orientation angle $\alpha_{\hat{\theta}_{P O L}}$. Right: RMSE vs. threshold of $\alpha_{\hat{\theta}_{P O L}}$. 

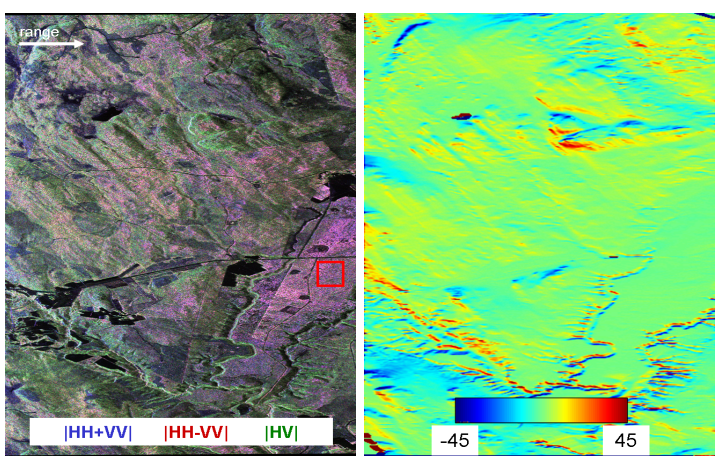

Fig. 4. Left: Pauli color-coded image of the test site of Krycklan. Right: LIDAR DTM-derived orientation angle.

\subsection{Boreal Forest With Topographic Variations}

The second test site is situated in Krycklan, Northern Sweden, and consists of a boreal forest over terrain with strong topographic variations. Figure 4 illustrates the Pauli color-coded image of the scene on the left hand side and on the right hand side the orientation angle in the range of -45 to +45 deg derived from a LIDAR digital terrain model (DTM) that is used as reference data.

The POLSAR-estimated orientation angle depicted in Figure 5 agrees very well with the LIDAR-derived orientation angle. It can be observed that the phase estimate is rather noisy over regions with high orientation angle variations (see Figure 5 on the right hand side). These areas are related to severely changing topography. The histogram of the POLSAR-derived orientation angle variations $\alpha_{\hat{\theta}_{P O L}}$ (Figure 6 on the top) shows that most of the samples possess rather low variations with values greater than 0.90 .

The RMSE over the whole image is at $10.4 \mathrm{deg}$ with a bias of -2.1 . When varying the variation threshold between 0 and $\frac{149}{151}$ (Figure 6 in the middle), the RMSE diminishes to approximately $5 \mathrm{deg}$. The lower the POLSAR-estimated orientation angle variations, the more precise the orientation angle estimate.

To examine the impact of the topography, the RMSE is calculated for all the samples whose LIDAR-derived orientation angle $\hat{\theta}_{\text {LIDAR }}$ is less than a given threshold thrsh $\hat{\theta}_{\mathrm{LIDAR}}$, i.e., with

$$
\hat{\theta}_{\mathrm{LIDAR}} \leq \operatorname{thrsh}_{\hat{\theta}_{\mathrm{LIDAR}}} .
$$

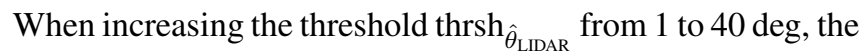
RMSE rises from approximately 5.6 to 9 deg as depicted in Figure 6 on the bottom. The lower the topographic variations in azimuth, the better the POLSAR-derived orientation angle estimates.

For a homogeneous forested area (see red square in Figure 4), the RMSE can be lowered to around 2.6 deg with a bias of -2.1 .

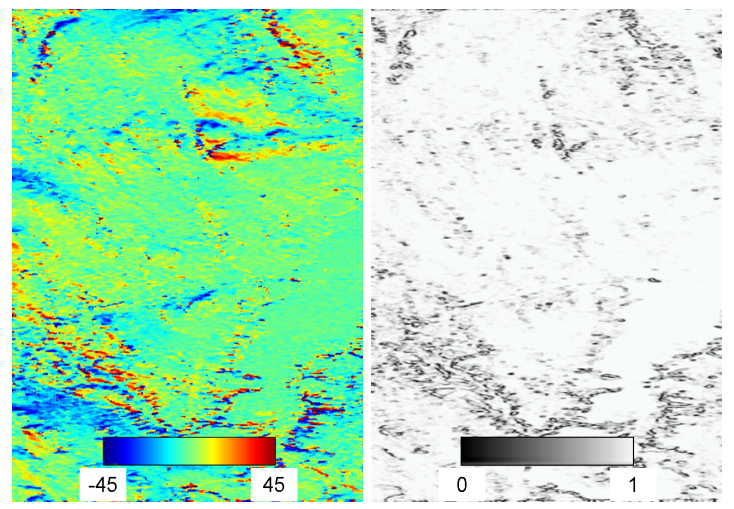

Fig. 5. Left: POLSAR-derived orientation angle over the test site of Krycklan. Right: Variations of the POLSAR-derived orientation angle $\alpha_{\hat{\theta}_{P O L}}$.
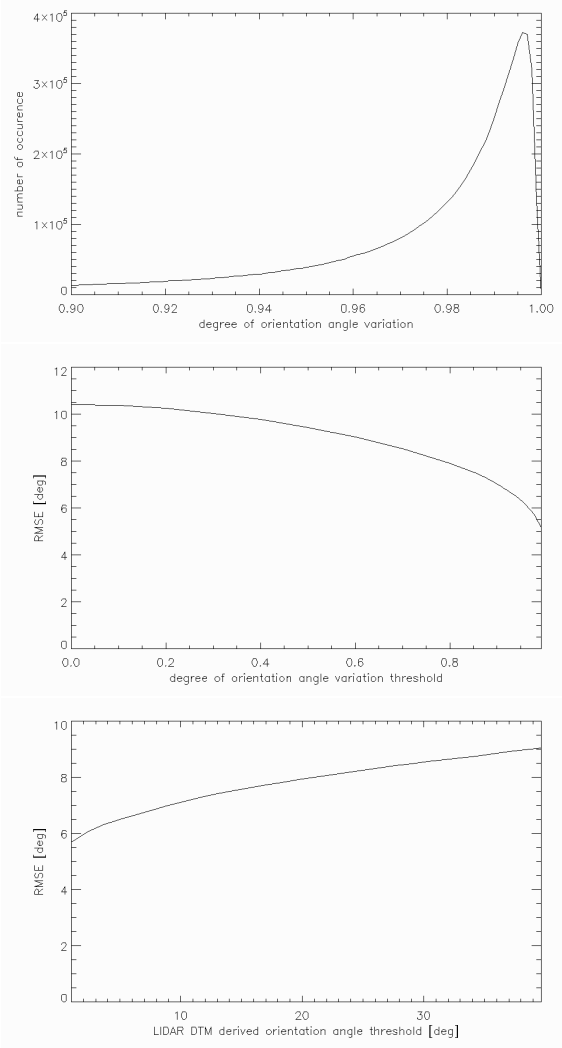

Fig. 6. Top: Histogram of variations of the POLSAR-derived orientation angle $\alpha_{\hat{\theta}_{P O L}}$. Middle: RMSE vs. threshold of $\alpha_{\hat{\theta}_{P O L}}$. Bottom: RMSE vs. DTM-derived orientation angle. 


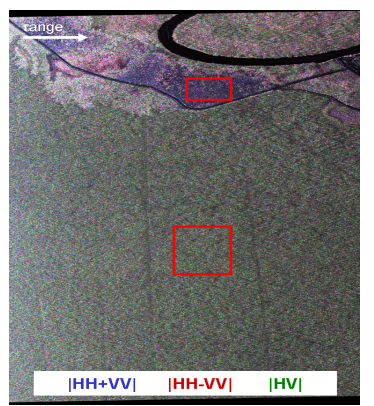

Fig. 7. Pauli color-coded image of the test site of Mawas.

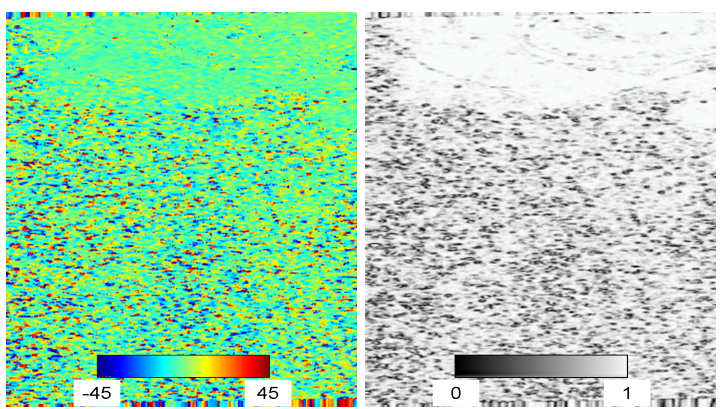

Fig. 8. Left: POLSAR-derived orientation angle over the test site of Mawas. Right: Variations of the POLSAR-derived orientation angle $\alpha_{\hat{\theta}_{P O L}}$.

\subsection{Tropical Forest Over Flat Terrain}

The Mawas test site in Indonesia includes a tropical forest over almost flat terrain. Figure 7 shows the Pauli color-coded image of the scene consisting largely of forested areas and a river and bare soils in the top part.

The orientation angle estimate shown in Figure 8 on the left hand side is rather strongly affected by noise, in particular over the forested areas. Although the terrain is almost flat, the variations of the POLSAR-estimated orientation angle over forested terrain are quite large as can be appreciated in Figure 8 on the right hand side and in the histogram of the variation parameter $\alpha_{\hat{\theta}_{P O L}}$ in Figure 9 on the left hand side. This is a clear indication that the orientation angle estimation accuracy is deteriorated by the dense vegetation.

The RMSE over the entire image is at 12.4 deg with a bias of -0.9 deg. When the variation of the POLSAR-derived orientation angle decreases, the RMSE diminshes to approximately 3 deg (see Figure 9 on the right hand side).

To examine the impact of the vegetation on the estimation performance, the homogeneous area including bare soil has been chosen as illustrated by the red square in Figure 7. In this area, the RMSE is approximately $3.7 \mathrm{deg}$ with a bias of $-1.7 \mathrm{deg}$. In contrast, in the homogeneous forested region encapsulated in the red square, the RMSE reaches a value of $15.4 \mathrm{deg}$ with a bias of $-2.7 \mathrm{deg}$.
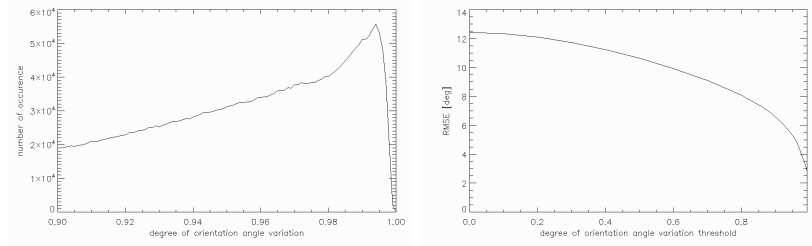

Fig. 9. Left: Histogram of variations of the POLSAR-derived orientation angle $\alpha_{\hat{\theta}_{P O L}}$. Right: RMSE vs. threshold of $\alpha_{\hat{\theta}_{P O L}}$.

\section{CONCLUSION}

In this paper, the performance of the circular polarization method for orientation angle estimation over forested scenes has been investigated by comparing the P-band POLSARderived orientation angles with the LIDAR DTM-derived orientation angles.

For boreal forests, the orientation angle estimation accuracy is rather high and degrades only slightly over forested areas compared with areas including bare soils. The estimation performance seems to be better over flat terrain than over areas having large topographic variations. For tropical forests, the estimation accuracy deteriorates rather significantly, even over flat terrain.

These investigations should be refined by separating the impacts of topography, vegetation, and homogeneity of the scene, e.g. by using polarimetric decomposition and classification techniques.

\section{REFERENCES}

[1] K. Scipal et al., "The biomass earth explorer mission candidate - status and results from phase-a activities," in Proceedings of POL-InSAR 2011, Jan. 2011.

[2] K. Scipal et al., "The biomass mission - an esa earth explorer candidate to measure the biomass of the earth's forests," in Proceedings of IGARSS 2010, July 2010.

[3] J. S. Kim, A. Danklmayer, and K. Papathanassiou, "Correction of ionospheric distortions in low frequency interferometric sar data," in Proceedings of IGARSS 2011, July 2011.

[4] J.-S. Lee, D. L. Schuler, and T. L. Ainsworth, "Polarimetric sar data compensation for terrain azimuth slope variation," IEEE Trans. Geoscience Remote Sensing, vol. 38, no. 5, pp. 2153-2163, Sept. 2000.

[5] J.-S. Lee, D. L. Schuler, T. L. Ainsworth, E. Krogager, D. Kasilingam, and W.-M. Boerner, "On the estimation of radar polarization orientation shifts induced by terrain slopes," IEEE Trans. Geoscience Remote Sensing, vol. 40, no. 1, pp. 30-41, Jan. 2002.

[6] D. L. Schuler, J.-S. Lee, and G. De Grandi, "Measurement of topography using polarimetric sar images," IEEE Trans. Geoscience Remote Sensing, vol. 34, no. 5, pp. 1266-1277, Sept. 1996.

[7] D. L. Schuler, J.-S. Lee, T. L. Ainsworth, and M. R. Grunes, "Terrain topography measurement using multipass polarimetric synthetic aperture radar data," Radio Science, vol. 35, no. 3, pp. 813-832, May-June 2000.

[8] H. Kimura, K. P. Papathanassiou, and I. Hajnsek, "Polarization orientation angle effects in urban areas on sar data," in Proceedings of IGARSS 2005, July 2005. 